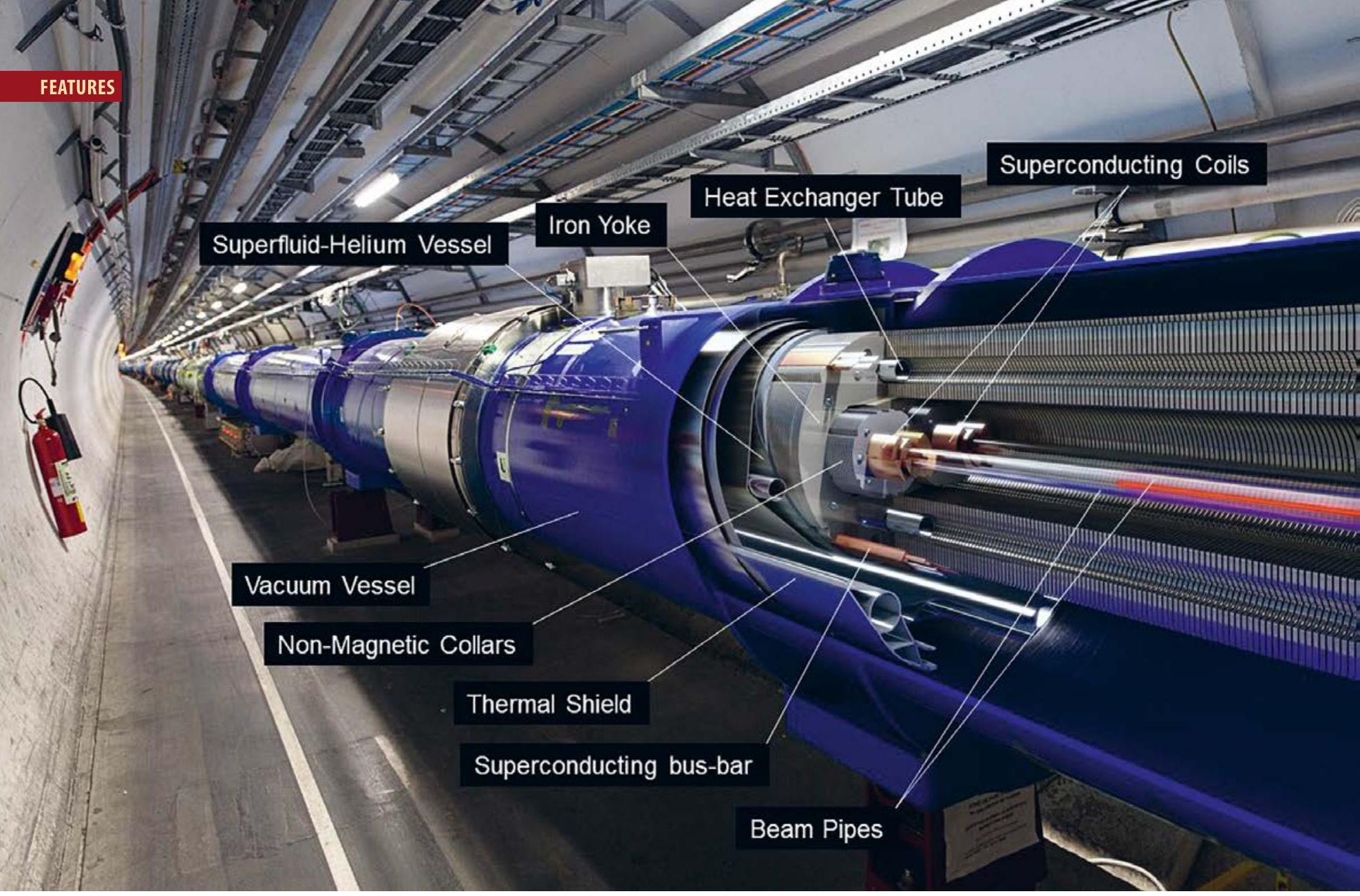

\title{
QUANTUM FLUIDS AT WORK: SUPERCONDUCTIVITY AND SUPERFLUID HELIUM AT THE LARGE HADRON COLLIDER
}

I Philippe Lebrun ${ }^{1}$ and Laurent Tavian ${ }^{2}$ - DOI: https://doi.org/10.1051/epn/2022108 I 'European Scientific Institute, Archamps (France) $-{ }^{2}$ CERN, Geneva (Switzerland)

The Large Hadron Collider (LHC) at CERN makes use of superconductivity and superfluid helium cryogenics on an unprecedented scale. We review the application of these technologies to the particle collider and explain how they contribute to its unique performance.

\footnotetext{
$\triangle$ FIG. 1:

3-D cut of

a twin-dipole cryomagnet in the LHC tunnel

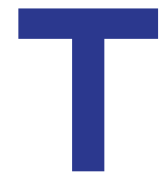
he Large Hadron Collider (LHC) is a $26.7 \mathrm{~km}$ circumference, high-energy proton and ion collider [1] in operation at CERN to serve the world's physics community. The LHC is also the single largest application of superconductivity and helium cryogenics to date, with its 8310 superconducting magnets of all types, most of which are operated at $1.9 \mathrm{~K}$ in some 100 tons of superfluid helium.
}

High-field magnets using low-temperature superconductors

Superconductivity and helium cryogenics have become key enabling technologies for high-energy accelerators over the last decades [2]. For a given beam energy, the diameter of a circular accelerator scales inversely with the field in the bending magnets: making the latter superconducting thus renders the machine more compact, 
allowing substantial savings in real estate and infrastructure. Moreover, compactness also reduces the beam stored energy for a given beam intensity, an important issue in high-energy, high-luminosity machines. Finally, superconductivity is also a means of reducing power consumption and thus operating costs of accelerators through two compounding processes: by enabling to make them smaller (the compactness argument above), and by reducing power per unit length of electromagnet. The power consumption of a superconducting synchrotron is essentially that of cryogenic refrigeration, which scales with the circumference of the machine irrespective of the field in the magnets.

The main technological stake of the LHC was the development, industrialization and production of 1232 superconducting dipoles with a field of $8.3 \mathrm{~T}$, 400 superconducting quadrupoles with a gradient of $223{\mathrm{~T} . \mathrm{m}^{-1}}^{1}$, and several thousand other superconducting magnets for correcting main field errors, tuning beam parameters and bringing beams into collision at high luminosity [3]. All these magnets were manufactured by industry and reproducibly produce field of the correct strength and uniformity with a precision of up to $10^{-4}$.

The main dipoles (Figure 1) feature twin apertures with equal and opposite fields in order to bend the two counter-rotating proton or ion beams along parallel paths. Two identical sets of coils are assembled in a common mechanical and magnetic structure, housed in a single cryostat. This solution is both compact in terms of transverse space occupancy and efficient, as the stray field of one aperture, channeled by the magnetic yoke, contributes to the field in the neighboring one. The coils in each aperture are wound with Rutherford-type Nb-Ti cable, in two layers with current density grading, following a "cos $\theta$ " geometry. The enormous electromagnetic forces that tend to open the structure when the magnet is powered are reacted by stiff collars of non-magnetic austenitic steel, resting on the magnetic steel yoke. The whole assembly is contained in an austenitic stainless steel pressure vessel that acts as helium enclosure.

The decrease in critical current of superconductors as the magnetic field increases restricts their use for high-field applications. This strongly limits the use of the well-known $\mathrm{Nb}-\mathrm{Ti}$ alloys in normal boiling helium at $4.2 \mathrm{~K}$. More advanced superconductors, such as $\mathrm{Nb}_{3}$ Sn were $\bullet \bullet \bullet$

- FIG. 2: A 13 kA current lead using high-Tc superconductors

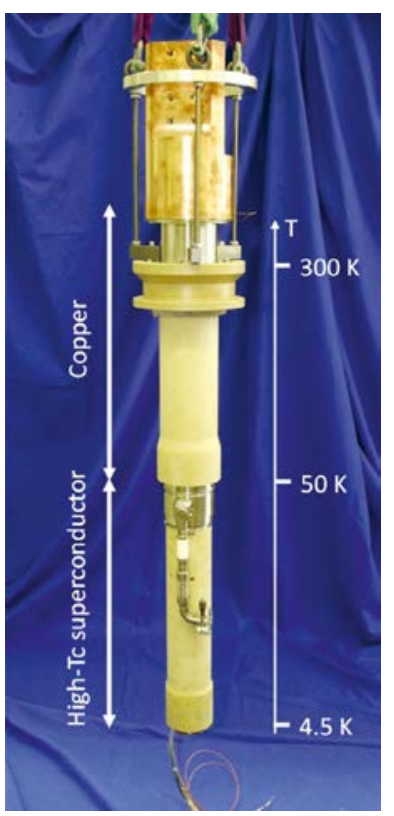

\section{THYR $\triangle \mathrm{CONT}$

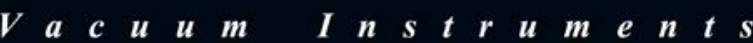

\section{Passive UHV Ion Gauge Controller}

We are pleased to announce that Thyracont has taken over the range of PVCuni / PVCduo ion gauge and vacuum system controllers from EpiMax Ltd. For more details on this, visit: http://www.epimax.com/products/pvcuniduo

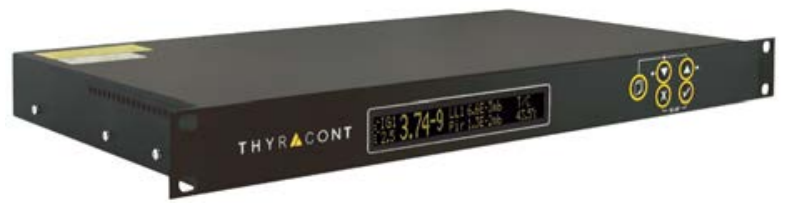

The PVC is an lon Gauge and Vacuum System Controller for passive UHV hot cathode ion gauges. It is available in different combinations with UHV ion gauges and various plug-in modules for operation of additional passive Pirani sensors VSP521/VSP841 or active 0-10V vacuum transducers.

An integrated thermocouple multistep bake-out control as well as MBE beam flux monitoring are supported by the PVC controller. Suitable hot cathode ion gauges and accessories like thermocouple temperature sensors for bake-out control or gauge cables are also available from Thyracont.

\section{Advantages and Possibilities:}

- $1 x$ UHV or $2 x$ UHV ion gauge

- Pirani gauges or active 0-10V transducers

- Intuitive menu-driven operation

- Auto ranging emission, or 11 manual emission settings

- Pressure measurement during degas, ramped power degas

- Integrated, interlocked, mulitstep bake-out controller

.7 output trips and 4 digital inputs

- Measurements displayed in pressure units or alternatively as direct ion current (emission corrected) for beam flux monitoring (BFM)

- MODBUS and ASClllbased QueBUS protocols

$\cdot 2 \times 0-10 V$ analog outputs

\section{For more information, contact:}

Thyracont Vacuum Instrument

https://thyracont-vacuum.com/en

+49851 95986-0 


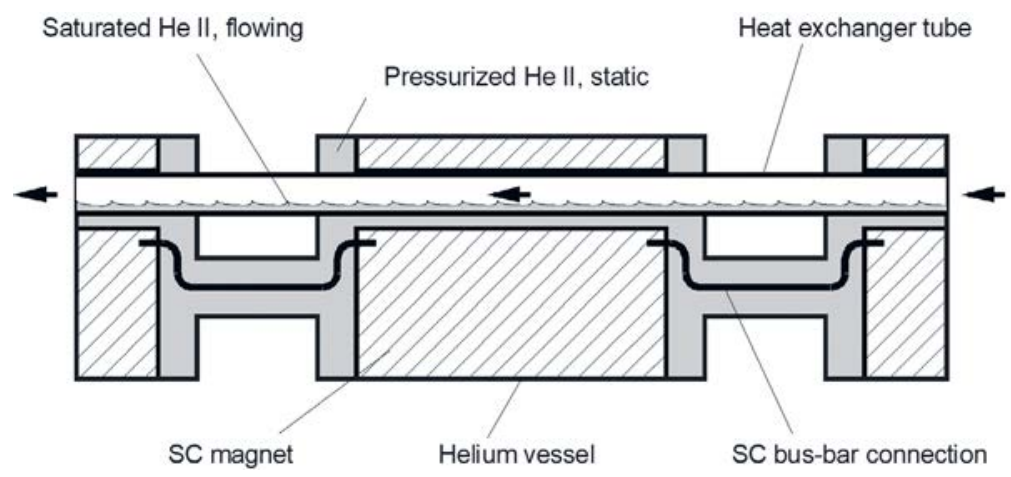

$\triangle$ FIG.3: found inadequate at the time, in view of their difficult Principle of the $\mathrm{LHC}$ superfluid helium (He II) cooling scheme technological implementation, limited industrial availability and high cost. CERN therefore decided to base the LHC project on Nb-Ti operated in superfluid helium at $1.9 \mathrm{~K}$. At this lower temperature, the alloy exhibits sufficient current-carrying capacity to produce fields up to about $10 \mathrm{~T}$, while superfluid helium maintains large enough thermal conductivity up to the lambda point. Superfluid helium, which has become a technical coolant for superconducting magnets following the pioneering work of G. Bon Mardion et al. [4] in Grenoble, was used for the first time in a large particle accelerator.

\section{High-temperature superconductors for high current transport}

Powering the 1720 electrical circuits of the LHC magnets requires to bring currents ranging from $60 \mathrm{~A}$ to $13 \mathrm{kA}$ from room temperature into the cryogenic environment. This is conventionally done by "current leads", i.e. copper conductors cooled by escaping helium vapor that intercepts the largest fraction of the conductive and Joule heat loads before they reach the liquid helium bath. Still, the remaining heat in-leak about 1.1 W.kA ${ }^{-1}$ for optimized metallic current leads would result in a prohibitively high load for the whole machine. The emergence of practical wires and ribbons made of high-Tc superconductors has made it possible to design, test and validate their use in the lower section of current leads, where they reduce signifi-

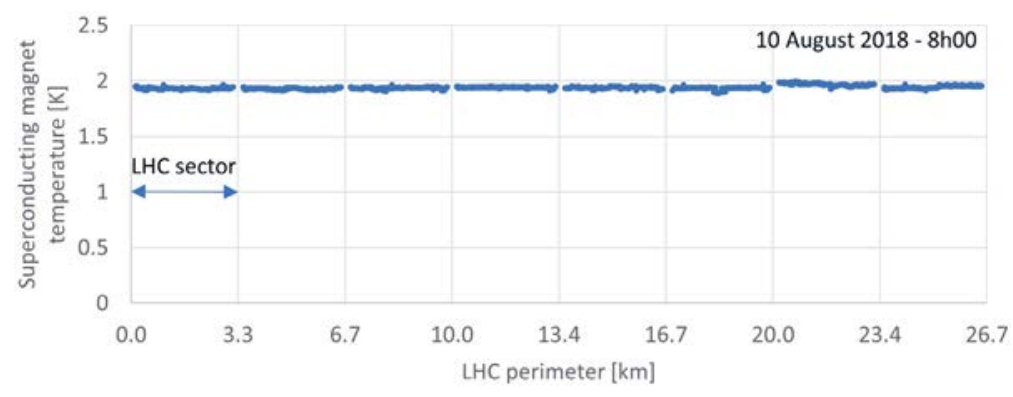

1182 high-Tc current leads equipping the high-current circuits of LHC (Figure 2) were produced by industry and are smoothly operating in the machine [5]. This represents the largest high-current application of high-Tc superconductors to-date.

\section{Superfluid helium as a cooling medium}

The main reason for superfluid helium cooling of the LHC magnets is the lower temperature that extends the operating range of the $\mathrm{Nb}$-Ti superconductor. However, the rapid drop in specific heat of the superconducting cable at low temperature also requires taking advantage of the very peculiar transport properties of superfluid helium for thermal stabilization, heat extraction from the magnet windings and heat transport to the cold source. With its low viscosity, superfluid helium can permeate the windings and buffer thermal transients thanks to its high specific heat -2000 times that of the conductor per unit volume. The excellent thermal conductivity of the fluid - peaking at 1000 times that of oxygen-free high-conductivity (OFHC) copper at $1.9 \mathrm{~K}$ for moderate heat flux - enables it to conduct heat without mass transport. In order to benefit from these unique properties, the electrical insulation of the cable must exhibit sufficient porosity and percolation, while preserving its main functions of mechanical resistance and dielectric strength. A multilayer wrapping of polyimide film with partial overlap meets these conflicting requirements.

The LHC magnets operate in static baths of pressurized superfluid helium close to atmospheric pressure. This high-conductivity mono-phase liquid is continuously cooled by heat exchange with saturated two-phase helium flowing in a $100 \mathrm{~m}$-long heat exchanger tube extending over the length of the magnet string (Figure 3). The latent heat of vaporization of the flowing helium eventually absorbs the deposited heat quasi-isothermally. Other benefits of this cooling scheme are the absence of convective flow in normal operation and corresponding pumps, the limited transverse space it occupies in the magnet cross-section, the capacity to absorb magnet resistive transitions and to limit their propagation [6]. Figure 4 shows the measured temperature profile around the LHC.

In view of the low saturation pressure of helium at $1.8 \mathrm{~K}$, refrigeration by vapor compression requires a pressure ratio of 80 to bring the helium back up to atmospheric pressure. To limit volume flow-rate and hence size of machinery, the large mass flow-rate in a high-power refrigerator must be processed at its highest density, i.e. cold. This calls for contact-less, vane-less, non-lubricated compressors of the hydrodynamic type [7]. The LHC uses eight $1.8 \mathrm{~K}$ refrigeration units, each with a refrigeration power of $2.4 \mathrm{~kW}$, based on multistage axial-centrifugal cold compressors operating at high rotational speed on active magnetic bearings (Figure 5). This technology developed by specialized industry following 
CERN's specifications was validated in the laboratory, with a measured overall coefficient of performance below 950 (ratio of electrical power to cooling power at $1.8 \mathrm{~K}$ ).

\section{Outlook}

All the technologies developed for the LHC are used, in enhanced form, for the High-Luminosity LHC (HLLHC) project now under construction: $1.2 \mathrm{~km}$ of the LHC collider will be upgraded, mainly in the regions close to the two large detectors, with the main objective to increase by a factor 10 the integrated number of collisions. New final-focus, large-aperture quadrupoles with a gradient of $948 \mathrm{~T} . \mathrm{m}^{-1}$ have been developed using advanced $\mathrm{Nb}_{3} \mathrm{Sn}$ superconductors. High-energy collision debris escaping the detectors will deposit a large fraction of their energy - a factor 5 increase with respect to the LHC - in the helium enclosure of these new quadrupole magnets cooled at $1.9 \mathrm{~K}$. The corresponding heat load will be extracted by two oversized heat exchanger tubes operating in parallel. Due to the high level of radiation close to the collision points, the magnet power converters and the high-Tc superconductor current leads are now located in protected areas at a distance of about $100 \mathrm{~m}$ from the machine tunnel. DC superconducting cables based on a novel concept using $\mathrm{MgB}_{2}$ operated

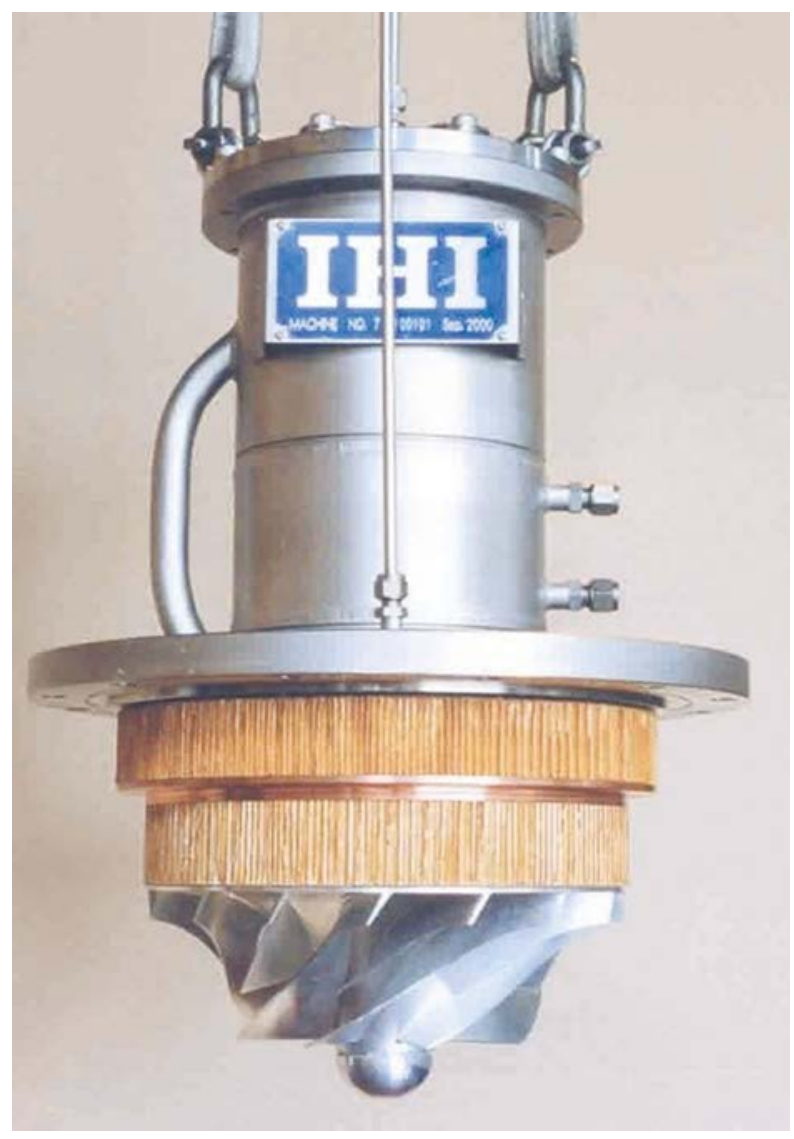

4FIG. 5: An axial-centrifugal cold helium compressor

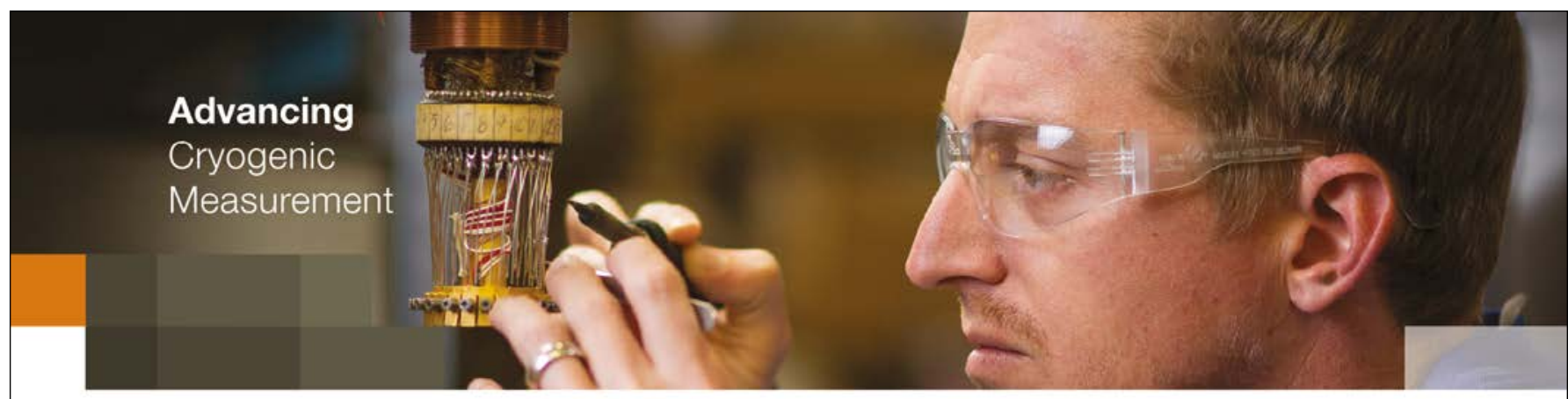

Precision cryogenic measurement solutions for your physics applications, including:

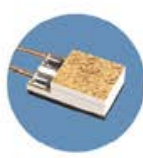

Temperature Sensors

Cernox ${ }^{\circledast}$ RTDs, diodes, and specialty types *

$<10 \mathrm{mK}$ to over $1500 \mathrm{~K}$ - flight-qualified versions .

wide selection of mounting packages available

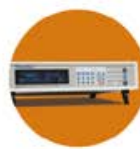

Temperature Controllers \& Monitors

Purpose built for cryogenic sensor measurement . current reversal eliminates thermal EMF offsets. up to $100 \mathrm{~W}$ of PID control $\cdot$ LabVIEW ${ }^{\text {Tu }}$ drivers available

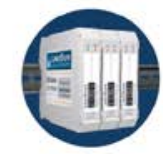

Sensor Input Modules

For monitoring in PLC-based applications . easily monitor temperatures from $1 \mathrm{~K}$ to $800 \mathrm{~K}$. support long cable connections to sensors

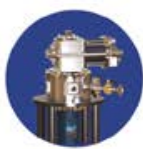

Laboratory Cryogenic Solutions

$\mathrm{LN}_{2}$, LHe, and closed-cycle refrigerator cryostats • cooling for ultra-high vacuum environments • application-specific and customized solutions

\section{Lake Shore} CRYOTRONICS

ADVANCING SCIENCE 614.891.2243 | www.lakeshore.com
To learn more about our products and services, contact sales@lakeshore.com 


\section{$9 \mathrm{GHz}$ mixer-free \\ qubit control and readout}

Introducing Presto:

A microwave rack-in-a-box to control multi-qubit chips. Qubit rotation, readout, coupling, DC bias - on 16 channels - and para-amp pumps.

No mixers, no microwave generators, just Direct Digital Synthesis up to $9 \mathrm{GHz}$.
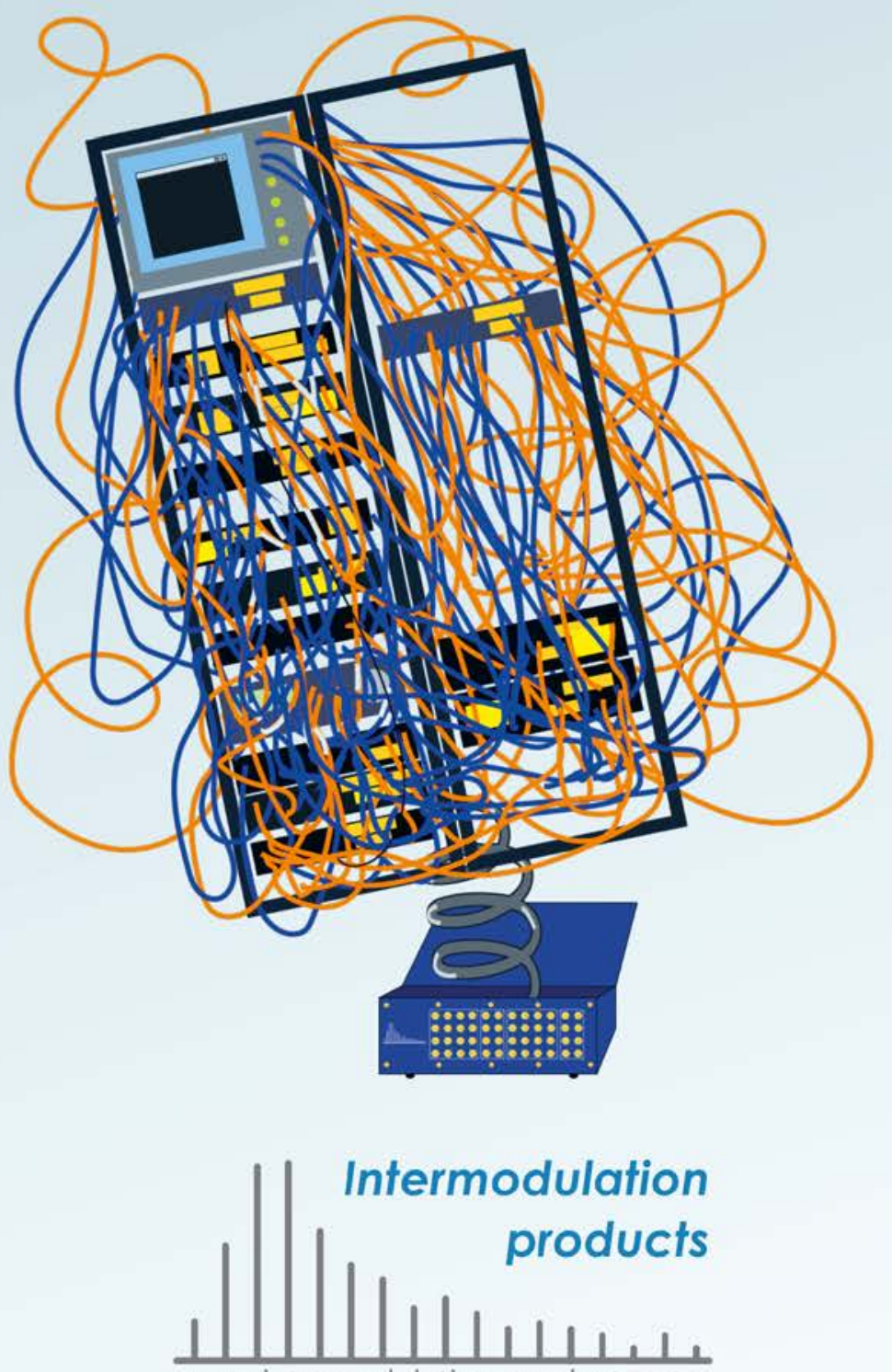

below $20 \mathrm{~K}$ have been developed to transport up to $120 \mathrm{kA}$ to the magnets. A novel superconducting RF system consisting of eight cavities per beam for transverse deflection (a.k.a. "crab" cavities) will compensate the geometric loss in luminosity due to the finite crossing angle and extreme focusing of the bunches in the HL-LHC.

As concerns future initiatives, the 2020 Update of the European Strategy for Particle Physics [8] has recommended as long-term priority the operation of a proton-proton collider at the highest achievable energy. Consequently, Europe together with its international partners is investigating the technical and financial feasibility of a Future Circular Collider (FCC) with a centre-of-mass energy of at least $100 \mathrm{TeV}$, located at CERN in a new 100-km circumference tunnel. This initiative will require to ramp up the R\&D effort on several advanced accelerator technologies, in particular high-field superconducting magnets including high-temperature superconductors.

\section{About the Authors}

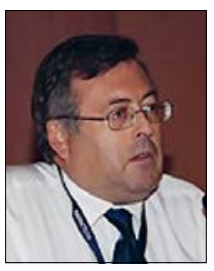

Philippe Lebrun. With a background in superconducting magnets and cryogenics, Philippe Lebrun led CERN’s “Accelerator Technology" department during the construction of the LHC. He has received several prizes in the field of cryogenics, including the Kamerlingh Onnes Medal, the Samuel Collins Award and the Kurt Mendelssohn Award.

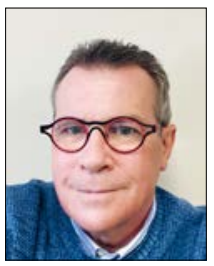

Laurent Tavian works in the Accelerator and Technology Sector at CERN, where heled the Cryogenics Group for 14 years. An engineer by training, he has contributed to the large superfluid helium cryogenic systems of the Tore Supra Tokamak and of the LHC.

\section{References}

[1] O. Brüning, P. Collier, Ph. Lebrun, S. Myers, R. Ostojic, J. Poole, P. Proudlock ed., LHC design report, CERN-2004-003 (2004)

[2] M. Wilson, Superconductivity and accelerators: the good companions, IEEE Trans. Appl. Superconductivity 9, 111 (1999)

[3] L. Rossi, Experience with LHC magnets, from prototyping to largescale industrial production, Proc. EPAC 2004 Lucerne, 118 (2004), JACoW web site

[4] G. Bon Mardion, G. Claudet \& J. C. Vallier, Superfluid helium bath for superconducting magnets, Proc. ICEC6, IPC Science \& Technology Press, Guildford (1976)

[5] A. Ballarino, High-temperature superconducting current leads for the Large Hadron Collider, IEEE Trans. Appl. Superconductivity 9, 523 (1999)

[6] M. Chorowski, Ph. Lebrun, L. Serio \& R. van Weelderen, Thermohydraulics of quenches and helium recovery in the LHC prototype magnet strings, Cryogenics 38, 533 (1998)

[7] Ph. Lebrun \& L. Tavian, Cooling with superfluid helium, in Proc. CAS "Superconductivity for accelerators", CERN-2014-005, 453 (2014)

[8] European Strategy Group, 2020 Update of the European Strategy for Particle Physics, https://europeanstrategyupdate.web.cern.ch 